\title{
Comunidad Parasitaria de Cabinza Isacia conceptionis (Cuvier \& Valenciennes, 1830) (Perciformes: Haemulidae) en la Zona de Chorrillos, Lima, Perú
}

\author{
Parasite Community of Cabinza Grunt Isacia conceptionis (Cuvier \& Valenciennes, \\ 1830) (Perciformes: Haemulidae) in the Area of Chorrillos, Lima, Peru
}

José Iannacone ${ }^{1,3,4}$, Lorena Alvariño ${ }^{1}$, Jhon Chero², Gloria Sáez ${ }^{2}$

\section{Resumen}

\begin{abstract}
El objetivo del presente trabajo fue evaluar la comunidad parasitaria de la cabinza Isacia conceptionis (Cuvier y Valenciennes, 1830) (Haemulidae) $(\mathrm{n}=123)$, procedente del Terminal Pesquero de Chorrillos, Lima, Perú, entre diciembre de 2010 y marzo de 2011. Los parásitos se fijaron, preservaron y cuantificaron mediante procedimientos estandarizados. Se colectaron 95 parásitos de siete especies, con una abundancia media total de 0.77 . El promedio de la riqueza de especies fue 0.46 . Los cuatro parásitos con mayor prevalencia y abundancia media fueron: Choricotyle isaciencis Oliva, González, Rus \& Luque, 2009, Corynosoma obtuscens (Lincicome, 1943), Proleptus carvajali Fernández y Villalba, 1985, y Clavella simplex Castro y Baeza, 1985. Los parásitos de I. conceptionis señalan que los tres índices de agregación [Dispersión (ID), Discrepancia de Poulin (D) y Binomial Negativa (k)] mostraron mayormente una distribución agregada. La prevalencia de $C$. isaciencis estuvo correlacionada con la longitud total del pez hospedero. En relación al $\mathrm{k}_{\mathrm{n}}$ (factor de condición relativo), no se observó que los peces parasitados con C. obtuscens, C. isaciencis, ectoparásitos y endoparásitos presentasen valores más altos en comparación con los peces no parasitados. El índice gonadosomático (IGS) no se relacionó con ninguno de los índices de ecología parasitaria. Los índices de equidad de la parasitofauna mostraron valores relativamente altos y, en cambio, los índices de dominancia fueron bajos. Los índices de interactividad $\left(\mathrm{CC}_{50}\right)$ de ectoparásitos y endoparásitos indican que las comunidades son aisladas.
\end{abstract}

Palabras clave: Choricotyle; Corynosoma; ecología parasitaria; Isacia

${ }^{1}$ Laboratorio de Ecofisiología Animal (LEFA), ${ }^{2}$ Laboratorio de Parasitología, Facultad de Ciencias Naturales y Matemática (FCNNM), Universidad Nacional Federico Villarreal (UNFV), Lima, Perú

${ }^{3}$ Facultad de Ciencias Biológicas, Universidad Ricardo Palma (URP), Lima, Perú

${ }^{4}$ E-mail:joseiannacone@gmail.com

Recibido: 12 de julio de 2014

Aceptado para publicación: 5 de noviembre de 2014 
The aim of this study was to evaluate the parasitic community of Cabinza grunt Isacia conceptionis (Cuvier y Valenciennes, 1830) (Haemulidae) ( $\mathrm{n}=123)$ from the Fish Market of Chorrillos, Lima, Peru during December 2010 to March 2011. Parasites were fixed, preserved and quantitated by standard procedures. A total of 95 parasites of seven species were collected with an average abundance of 0.77 . The average species richness was 0.46. The four most prevalent parasites and mean abundance were Choricotyle isaciencis Oliva, Gonzalez, Rus y Luque, 2009, Corynosoma obtuscens (Lincicome, 1943), Proleptus carvajali Fernández y Villalba, 1985, and Clavella simplex Castro y Baeza, 1985. Parasites of I. conceptionis indicate that the three indices of aggregation [Dispersion (ID), Discrepancy of Poulin (D) and Negative Binomial (k)] mostly showed an aggregated distribution. The prevalence of $C$. isaciencis correlated with the total length of the host fish. In relation to $\mathrm{k}_{\mathrm{n}}$ (relative condition factor), none of the fish parasitized by C. obtuscens, C. isaciencis, ectoparasites and endoparasites presented higher values compared with non-parasitized fish. The gonadosomatic index (GSI) was not associated with any of the indices of parasite ecology. Equity indices of parasitefauna showed relatively high values. Instead, dominance rates were low. Rates of interactivity $\left(\mathrm{CC}_{50}\right)$ of ectoparasites and endoparasites indicated that communities were isolated.

Key words: Choricotyle; Corynosoma; parasite ecology; Isacia

\section{INTRODUCCIÓN}

Varios parámetros del pez hospedero (características intrínsecas) y abióticas (extrínsecas) tienen un efecto en las comunidades parasitarias (Poulin, 2010). Estas últimas han sido empleadas como herramientas para discriminar poblaciones hospederas, interacciones en la cadena trófica e identificar ambientes contaminados (Centeno y Bashirullah, 2003). La talla y el sexo del pez hospedero son consideradas como variables ecológicas relevantes que se asocian con la oscilación de las comunidades parasitarias (Luque y Poulin, 2008; Bryan et al., 2012; Iannacone y Alvariño, 2013; Karling et al., 2013).

La cabinza Isacia conceptionis (Cuvier y Valenciennes, 1830) (Haemulidae) es una especie de pez asociada a ambientes arenosos-rocosos, cuya comercialización satisface mayormente el consumo humano di- recto, teniendo un desembarque en el Perú de 2745 t. Se distribuye desde la Isla Lobos de Afuera (Perú) hasta Talcahuano (Chile) (Chirichigno y Cornejo, 2001; González et al., 2001; IMARPE, 2014). I. conceptionis es una especie considerada ictiófaga en relación a su espectro trófico (Vargas et al., 1999). Los mayores niveles de desove se dan en la primavera y verano (Cortes y Aron, 2011).

Varios autores han registrado parásitos metazoos para I. conceptionis en la costa peruana-chilena (Morales et al., 1987; Luque y Farfán, 1991; Tantaleán y Huiza, 1994; Sarmiento et al., 1999; Gonzáles et al., 2001; Tantaleán et al., 2005; Kohn et al., 2007; Muñoz y Olmos, 2007, 2008; Cohen et al., 2013). Hasta la fecha, no existen trabajos en el Perú que analicen la fauna comunitaria de los parásitos de I. conceptionis procedentes de la costa central marina. El objetivo del presente trabajo fue evaluar la comunidad parasitaria de I. conceptionis procedente del Terminal Pesquero de Chorrillos, Lima, Perú. 


\section{Materiales y Métodos}

Se adquirieron 123 especímenes de «cabinza», I. conceptionis, entre diciembre de 2010 y marzo de 2011 en el Terminal Pesquero de Chorrillos, Lima, Perú. Los peces fueron identificados empleando las ilustraciones y claves taxonómicas de Chirichigno y Vélez (1998).

Se examinaron las branquias, cavidad bucal, estómago, intestino y cavidad celómica para la búsqueda de parásitos metazoos. Los platelmintos y acantocéfalos fueron colectados, fijados en solución AFA (alcohol al 70\% - formol - ácido acético glacial), preservados en alcohol al 70\%, coloreados con carmín acético de Semichon o tricrómica de Gomori, y montados en bálsamo de Canadá. Los nematodos fueron aislados, lavados en solución salina, fijados en alcohol caliente al 70\% y aclarados en una mezcla de alcohol-fenol.

La identificación taxonómica de los parásitos se basó en las descripciones originales de las taxas y, en algunos casos, de claves especializadas a nivel de especie (Tantaleán et al., 1982, 1992; Castro y Baeza, 1985; Palm, 1999; Kohn et al., 2007; Oliva et al., 2009; Cohen et al., 2013). Especímenes representativos de los parásitos encontrados en I. conceptionis fueron depositados en la Colección de Protozoos y Metazoos Parásitos de la Universidad Nacional Federico Villarreal (CPMP-UNFV) y en la colección de helmintología y de invertebrados menores del Museo de Historia Natural de la Universidad Nacional Mayor de San Marcos (MUSM- UNMSM).

En cada espécimen se determinó el sexo, peso corporal (W) en gramos y longitud total (LT) en centímetros. La LT de los peces se dividió en cuatro rangos de $3.0 \mathrm{~cm}$ cada uno mediante la regla de Sturges. Los rangos fueron 16.5-19.5 $(\mathrm{n}=15), 19.6-22.5$ $(\mathrm{n}=76), 22.6-25.5(\mathrm{n}=27)$ y $25.6-28.40(\mathrm{n}=5)$.
Se usó la prueba de t Student para determinar diferencias entre LT de machos y hembras, cumpliéndose previamente con el requisito de normalidad, con la prueba de Kolmogorov-Smirnov, y de homogeneidad de varianza, con la prueba de Levene.

Para caracterizar la infección de cada especie parásita, se determinaron los parámetros de ecología parasitaria, según Bush et al. (1997): prevalencia (P), intensidad media de infección (IM) y abundancia media de infección (AM). También se calculó la prevalencia y la abundancia total. El paquete estadístico Quantitative Parasitology v. 3.0 se usó para determinar estos valores y sus límites de confianza al 95\%. La P se calculó según el método exacto de Sterne, con más de 1000 réplicas ajustadas al método Ward. Para los intervalos de confianza al 95\% de IM y AM se emplearon 2000 réplicas bootstrap (Reiczigel, 2003). Asimismo, se calculó el índice de importancia específica (I) para cada especie parásita y para el total de parásitos, donde I = Prevalencia + (abundancia media $x$ 100) (Céspedes et al., 2011), con el fin de obtener un índice integrado de infección de ambos descriptores ecológicos.

La frecuencia de dominancia de cada taxa parásita se calculó como el número de veces que es dominante una taxa en todos los peces examinados. La frecuencia de dominancia relativa de cada taxa fue determinada como el número de individuos de una taxa sobre el número total de individuos de todas las taxas en la infracomunidad parasitaria (Rohde et al., 1995). Para el caso de las especies parásitas con prevalencias mayores del 10\% (Esch et al., 1990), se emplearon tres índices de agregación: Dispersión (ID), Discrepancia de Poulin (D) y Binomial Negativa (k) (Bliss y Fisher, 1953; Poulin, 1993; Bego y Von Zuben, 2010) (Cuadro 1). Quantitative Parasitology v. 3.0 se utilizó para el cálculo de estos índices y mostrar si los parásitos presentaban una distribución (a) contagiosa, agregada o conglomerada, (b) uniforme-regular o (c) aleatorizada, al azar o randomizada (Rózsa et al., 2000) (Cuadro 1). 
Cuadro 1. Índices de agregación e interpretación para evaluar parásitos de I. conceptionis provenientes del Terminal Pesquero de Chorrillos, Lima, Perú, empleando el paquete estadístico Quantitative Parasitology v. 3.0

\begin{tabular}{lcccc}
\hline \multirow{2}{*}{ Índice de agregación } & \multirow{2}{*}{ Acrónimo } & \multicolumn{3}{c}{ Distribución } \\
\cline { 3 - 5 } & & Uniforme & Agregado & Aleatorio \\
\hline Índice de dispersión & $\mathrm{ID}$ & $<1$ & $>1$ & 1 \\
Índice de discrepancia de Poulin & $\mathrm{D}$ & 0 & 1 & \pm 0.5 \\
Binomial Negativa & $\mathrm{K}$ & $>8$ & $<2$ & $2-8$ \\
\hline
\end{tabular}

El coeficiente de correlación de Pearson se usó para determinar la relación entre el tamaño del pez hospedero (LT) con la abundancia de cada parásito metazoa. El coeficiente de correlación de Spearman se empleó para determinar la relación entre la LT del hospedero y la $\mathrm{P}$ de cada parásito metazoa. Se aplicó la prueba $\mathrm{X}^{2}$ razón de verosimilitud para tablas de contingencia $2 \times 2$ para determinar el grado de dependencia entre el sexo del hospedero y la P. Para evaluar el efecto del sexo en la AM de infección parasitaria, se utilizó la prueba de t Student.

El factor de condición relativo $\left(\mathrm{K}_{\mathrm{n}}\right)$ fue obtenido a base del W y LT de I. conceptionis, a partir de la fórmula: $\mathrm{K}_{\mathrm{n}}={ }^{\mathrm{W}} / \mathrm{W}^{*}$, donde: $\mathrm{W}=$ peso de la cabinza $(\mathrm{g})$ y $\mathrm{W}^{*}=$ peso estimado por la ecuación de regresión lineal LT-W previamente calculada (Longart et al., 2011). Se usó la prueba de t Student para comparar los valores de $\mathrm{K}_{\mathrm{n}}$ entre los peces parasitados $\mathrm{y}$ no parasitados para las especies de helmintos más prevalentes (ectoparásitos, endoparásitos y en conjunto). El índice gonadosomático (IGS) se determinó como la siguiente relación: IGS $=[\mathrm{W}$ gónadas / (Wtotal Wgónadas) $] \times 100$. El IGS se correlacionó con la LT, abundancia total, prevalencia total, y con la abundancia de $C$. obtuscens y $C$. isaciencis.
En el cálculo de la diversidad parasitaria se usaron los siguientes índices: ShannonWiener $\left(\mathrm{H}^{\prime}\right)$, Equitabilidad de Pielou $\left(\mathrm{J}^{\prime}\right)$, Dominancia numérica de Berger-Parker $\left(\mathrm{I}_{\mathrm{B}-\mathrm{P}}\right)$ y Dominancia de Simpson (ë) (Moreno, 2001). Los índices de interactividad $\left(\mathrm{CC}_{50}\right)$ de ectoparásitos y endoparásitos (Poulin y Luque, 2003) se analizaron con el fin de determinar el porcentaje de hospederos que necesitan ser examinados para encontrar al $50 \%$ de especies parásitas, al ser ordenados primero los hospederos de las especies más pobres y luego a las especies más ricas, incluyendo los hospederos no infectados. Se empleó un estimador de riqueza (Chao-1) para estimar el número máximo de riqueza de parásitos a partir del esfuerzo de muestreo realizado y de la acumulación de nuevas taxa que van apareciendo en las cabinzas muestreadas (Bautista et al., 2013). Los dendrogramas se calcularon mediante el método Ward para comparar la similitud de los parásitos. El análisis de los índices de diversidad y del estimador de riqueza de parásitos se realizaron con la ayuda del programa PAST (Paleontological Statistics software) v. 2012 (2.16). El nivel de significancia fue evaluado a un alfa $=0.05$. Para el cálculo de las pruebas estadísticas descriptivas e inferenciales, se usó el paquete estadístico IBM SPSS Statistics 21.0 - 2012. 
Cuadro 2. Ubicación, prevalencia, intensidad y abundancia media de los parásitos de Isacia conceptionis adquiridos en el Terminal Pesquero de Chorrillos, Lima, Perú1, empleando el paquete estadístico Quantitative Parasitology v. 3.0

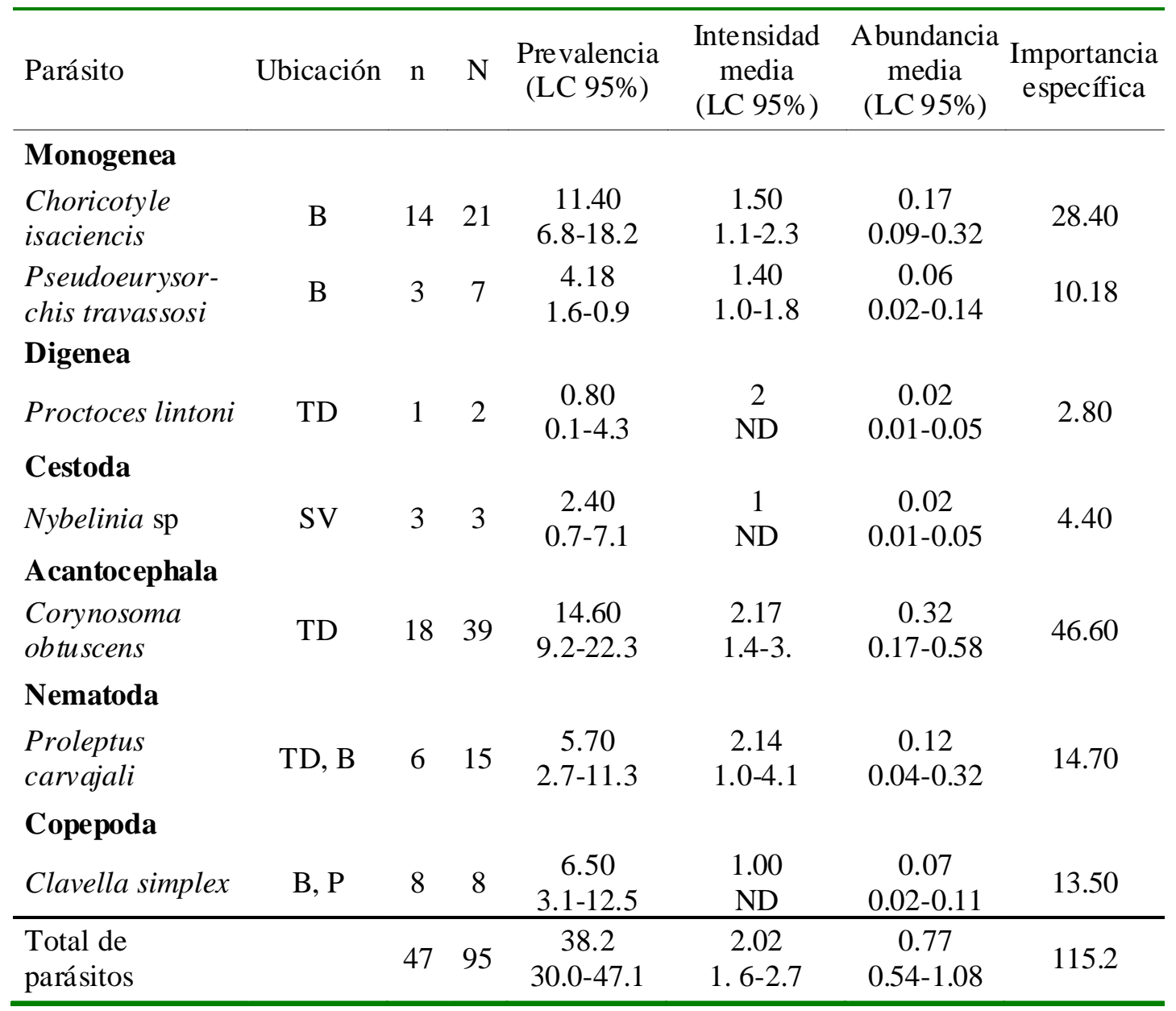

${ }^{1} \mathrm{~N}$ : Número total de parásitos; $\mathrm{n}$ : Número de hospederos infectados; B: branquias; $\mathrm{P}$ : piel; TD: tubo digestivo; SV: superficie visceral; ND: No determinado

\section{Resultados}

La LT varió entre 16.5 y $28.4 \mathrm{~cm}(21.7$ \pm 2.0 ), donde la LT de los machos ( $\mathrm{n}=59$ ) fue de $21.3 \pm 1.4 \mathrm{~cm}$ y de las hembras $(\mathrm{n}=64)$ fue de $22.1 \pm 2.3 \mathrm{~cm}$, habiendo diferencias significativas entre $\operatorname{sexos}(\mathrm{t}=2.49 ; \mathrm{p}=0.01)$.

Se colectaron 95 parásitos, con una abundancia media total de 0.77 . El promedio de la riqueza de especies fue $0.46 \pm 0.63$ ( 0 2). Del total de hospederos, 76 (28.6\%) no mostraron infección parasitaria, en tanto que $39(31.7 \%)$ y $8(6.5 \%)$ de hospederos estuvieron infectados con una y dos especies parásitas, respectivamente.

En el Cuadro 2 se muestra la ubicación, P, IM y AM de infección de las siete taxa de parásitos encontrados. Los cuatro parásitos con mayor importancia específica (prevalencia y abundancia media) fueron Choricotyle isaciencis Oliva, González, Rus y Luque, 2009, Corynosoma obtuscens Lincicome, 1943, Proleptus carvajali Fernández y 
Cuadro 3. Frecuencia de dominancia de los parásitos componentes de Isacia conceptionis adquiridos en el Terminal Pesquero de Chorrillos, Lima, Perú

\begin{tabular}{lccc}
\hline Parásito & $\begin{array}{c}\text { Frecuencia } \\
\text { de } \\
\text { dominancia }\end{array}$ & $\begin{array}{c}\text { Frecuencia de } \\
\text { dominancia de dos } \\
\text { especies }\end{array}$ & $\begin{array}{c}\text { Frecuencia de } \\
\text { dominancia } \\
\text { relativa }\end{array}$ \\
\hline Choricotyle isaciencis & 10 & 1 & $0.09 \pm 0.30$ \\
Pseudoeurysorchis travassosi & 2 & 2 & $0.02 \pm 0.10$ \\
Proctoces lintoni & 1 & 0 & $0.01 \pm 0.01$ \\
Nybelinia sp. & 3 & 0 & $0.02 \pm 0.20$ \\
Corynosoma obtuscens & 14 & 2 & $0.12 \pm 0.30$ \\
Proleptus carvajali & 6 & 2 & $0.04 \pm 0.20$ \\
Clavella simplex & 6 & 2 & $0.05 \pm 0.20$ \\
\hline
\end{tabular}

Cuadro 4. Índices de agregación para evaluar parásitos de Isacia conceptionis adquiridos del Terminal Pesquero de Chorrillos, Lima, Perú, empleando el paquete estadístico Quantitative Parasitology v. 3.0

\begin{tabular}{lccc}
\hline & \multicolumn{3}{c}{ Índices de agregación $^{1}$} \\
\cline { 2 - 4 } & $\mathrm{ID}$ & $\mathrm{D}$ & $\mathrm{k}$ \\
\hline Choricotyle isaciencis & 2.08 & 0.91 & 0.18 \\
Pseudoeurysorchis travassosi & 1.81 & 0.96 & $\mathrm{ND}^{2}$ \\
Proctoces lintoni & 2.00 & 0.98 & $\mathrm{ND}$ \\
Nybelinia sp & 0.98 & 0.97 & $\mathrm{ND}$ \\
Corynosoma obtuscens & 3.68 & 0.91 & 0.12 \\
Proleptus carvajali & 4.11 & 0.95 & 0.04 \\
Clavella simplex & 0.94 & 0.92 & $\mathrm{ND}$ \\
\hline Abundancia total & 3.07 & 0.76 & 0.48 \\
\hline
\end{tabular}

${ }^{1}$ ID: Varianza $\left(S^{2}\right) /$ abundancia media de infección; $D$ : Índice de discrepancia; $K=$ exponente de la Binomial Negativa

${ }^{2}$ No determina do

Villalba, 1985, y Clavella simplex Castro y Baeza, 1985. Los monogeneos y nematodos dominaron en número de especies $(\mathrm{n}=2)$ en cada grupo taxonómico. En porcentaje de individuos colectados, los endoparásitos dominaron $(62.1 \% ; \mathrm{n}=59)$ frente a los ectoparásitos $(37.9 \%$; $\mathrm{n}=36)$. La mayor frecuencia de do- minancia y de dominancia relativa fueron para el $C$. obtuscens y $C$. isaciencis (Cuadro 3).

El Cuadro 4 muestra los tres índices de agregación [Dispersión (ID), Discrepancia de Poulin (D) y Binomial Negativa (k)] para los parásitos encontrados que mostraron ma- 
Cuadro 5. Coeficientes de correlación (r) usados para evaluar la relación entre la longitud total de Isacia conceptionis versus la prevalencia y abundancia de los parásitos en peces adquiridos en el Terminal Pesquero de Chorrillos, Lima, Perú ${ }^{1}$

\begin{tabular}{lcccc}
\hline & $\begin{array}{c}\mathrm{r}^{*} \\
\text { (Spearman) }\end{array}$ & $\mathrm{p}$ & $\begin{array}{c}\mathrm{r}^{* *} \\
(\text { Pearson })\end{array}$ & $\mathrm{p}$ \\
\hline Choricotyle isaciencis & 1.00 & 0.001 & -0.01 & 0.91 \\
Corynosoma obtuscens & -0.80 & 0.20 & 0.04 & 0.62 \\
Prevalencia total & -0.40 & 0.60 & $\mathrm{NA}^{2}$ & $\mathrm{NA}$ \\
Abundancia total & $\mathrm{NA}$ & $\mathrm{NA}$ & -0.04 & 0.62 \\
\hline${ }^{1} \mathrm{p}=$ nivel de significancia; $\mathrm{r}$ =coeficiente de correlación; * \\
$=$ longitud total vs abundancia \\
${ }^{2}$ No aplica
\end{tabular}

Cuadro 6. Valores de la prueba de t student (t) y del estadístico Razón de Verosimilitud empleando Chi-cuadrado $\left(\mathrm{X}^{2}\right)$ en la evaluación de la relación entre sexo de Isacia conceptionis y la abundancia y prevalencia de infección de peces adquiridos en el Terminal Pesquero de Chorrillos, Lima, Perú ${ }^{1}$

\begin{tabular}{lcccc}
\hline Parásito & $\mathrm{t}^{*}$ & $\mathrm{p}$ & $\mathrm{X}^{2} * *$ & $\mathrm{P}$ \\
\hline Choricotyle isaciencis & 0.62 & 0.53 & 0.16 & 0.68 \\
Corynosoma obtuscens & 0.05 & 0.96 & 0.48 & 0.48 \\
Prevalencia total & $\mathrm{NA}^{2}$ & $\mathrm{NA}$ & 1.64 & 0.19 \\
Abundancia total & 0.51 & 0.60 & $\mathrm{NA}$ & $\mathrm{NA}$ \\
\hline
\end{tabular}

${ }^{1}$ nivel de significancia; $*$ = compara la abundancia media entre sexos; $* *=$ compara la prevalencia de infección entre sexos

${ }^{2}$ No aplica

Cuadro 7. Comparación entre los valores del factor de condición relativo $\left(\mathrm{k}_{\mathrm{n}}\right)$ entre parasitados y no parasitados más prevalentes de I. conceptionis adquiridos del Terminal Pesquero de Chorrillos, Lima, Perú

\begin{tabular}{lcccc}
\hline Parásito & $\begin{array}{c}\mathrm{K}_{\mathrm{n}} \\
\text { parasitados }\end{array}$ & $\begin{array}{c}\mathrm{K}_{\mathrm{n}} \text { no } \\
\text { parasitados }\end{array}$ & $\mathrm{t}$ & $\mathrm{P}$ \\
\hline Choricotyle isaciencis & 1.010 & 0.999 & 0.22 & 0.82 \\
Corynosoma obtuscens & 1.001 & 1.001 & 0.004 & 0.99 \\
Ectoparásitos & 0.954 & 1.012 & 1.53 & 0.12 \\
Endoparásitos & 1.012 & 0.996 & 0.42 & 0.67 \\
Todos los parásitos & 0.989 & 1.007 & 0.56 & 0.57 \\
\hline
\end{tabular}




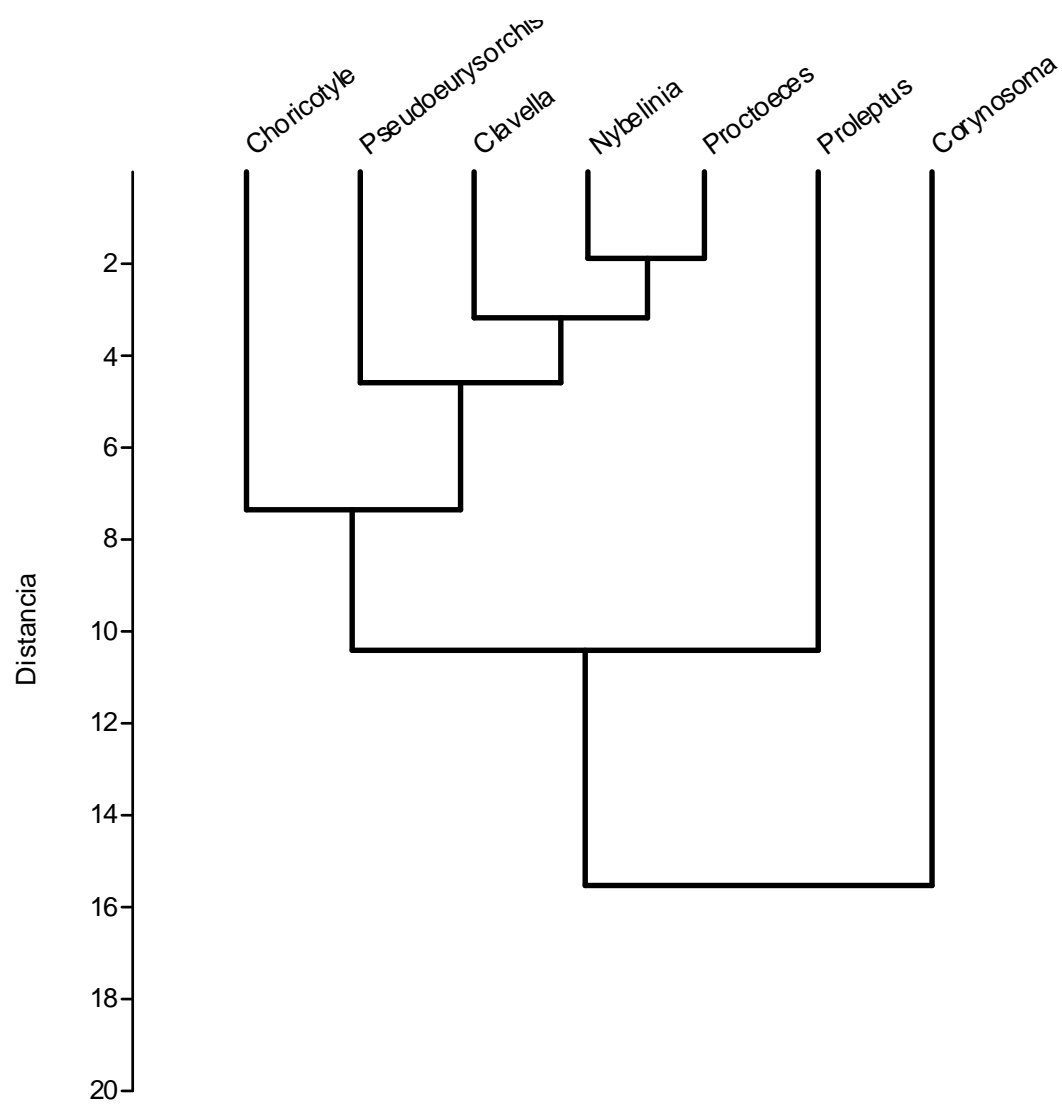

Figura 1. Dendrograma de similaridad mediante el método de Ward de asociación entre parásitos de Isacia conceptionis adquiridos en el Terminal Pesquero de Chorrillos, Lima, Perú (r=0.978)

Cuadro 8. Índice no paramétrico que estima el número de especies de parásitos a encontrarse, de diversidad alfa y de interactividad $\left(\mathrm{CC}_{50}\right)$ de la parasitofauna de Isacia conceptionis adquiridos en el Terminal Pesquero de Chorrillos, Lima, Perú

\begin{tabular}{lc}
\hline \multicolumn{1}{c}{ Índices } & Valores \\
\hline Chao-1 & 8 \\
Shannon-Wiener $\left(\mathrm{H}^{\prime}\right)$ & 1.64 \\
Equitabilidad de Pielou $\left(\mathrm{J}^{\prime}\right)$ & 0.79 \\
Simpson $(\lambda)$ & 0.25 \\
Berger-Parker $\left(\mathrm{I}_{\mathrm{B}-\mathrm{P}}\right)$ & 0.41 \\
Interactividad ectoparásitos $\left(\mathrm{CC}_{50}\right)$ & 79.78 \\
Interactividad endoparásitos $\left(\mathrm{CC}_{50}\right)$ & 78.97 \\
\hline
\end{tabular}

yormente una distribución agregada. Solo Proctoeces lintoni Siddiqi y Cable, 1960 y C. simplex según ID presentaron una distribución uniforme. No se determinó el tipo de distribución por la Binomial Negativa (k) en varios parásitos debido a la baja prevalencia observada.

El Cuadro 5 muestra que solamente la $\mathrm{P}$ de $C$. isaciencis estuvo correlacionada con la longitud total del hospedero. Asimismo, el sexo del pez no tuvo relación con la $\mathrm{P}$ y abundancia de infección (Cuadro 6). En relación con $\mathrm{k}_{\mathrm{n}}$, no se vio que los peces parasitados por $C$. obtuscens, $C$. isaciencis, ectoparásitos y endoparásitos presentaran valores más altos en comparación con los peces no parasitados (Cuadro 7). El $\mathrm{k}_{\mathrm{n}}$ no se correlacionó con la 
abundancia total, riqueza parasitaria e IGS (r $= \pm 0.003-0.014 ; \mathrm{p}=0.41-0.97)$. El IGS solo se correlacionó con la LT $(\mathrm{r}=0.37 ; \mathrm{p}=0.001)$, pero no se observó asociado con la abundancia total, prevalencia total, ni con la abundancia de $C$. obtuscens y $C$. isaciencis.

Los índices de diversidad alfa $\left(\mathrm{H}^{\mathrm{I}}\right.$ y $\left.\mathrm{J}^{\mathrm{I}}\right)$ de la parasitofauna de I. conceptionis mostraron valores relativamente altos (Cuadro 8). En cambio, los índices de dominancia $\left(\lambda \mathrm{I}_{\mathrm{B}-\mathrm{P}}\right)$ tuvieron valores bajos. Los índices de interactividad $\left(\mathrm{CC}_{50}\right)$ de ectoparásitos $\mathrm{y}$ endoparásitos mostraron valores bajos que muestran que las comunidades no son interactivas o son aisladas. El estimador no paramétrico Chao-1 indicó que no se requiere aumentar el esfuerzo de muestreo de los peces hospederos, pues se logró registrar ocho taxa durante el periodo evaluado y el valor de riqueza esperado de especies parásitas fue de 8 (Cuadro 8).

\section{Discusión}

En el presente estudio se observó el predominio de los endoparásitos sobre los ectoparásitos en I. conceptionis. La literatura señala que la dominancia de endoparásitos puede ser atribuida al comportamiento trófico por ser organismos principalmente ictiófagos-omnívoros, que incluyen una gran variedad de invertebrados acuáticos que pueden ser hospederos intermediarios en el ciclo de vida de varios helmintos (Vargas et al., 1999). La dominancia de endoparásitos se ha observado en el pez haemulido Orthopristis ruber (Cuvier, 1829) en el Golfo de Cariaco, Venezuela (Centeno et al., 2002), y para otras comunidades parasitarias en peces marinos de la costa del Perú (Iannacone et al., 2010b; Iannacone et al., 2012).

La mayor frecuencia de dominancia y de dominancia relativa fueron para el acantocéfalo $C$. obtuscens y el monogeneo C. isaciencis. Dentro de los ectoparásitos, el monogeneo $C$. isaciencis fue el más dominante, aunque con baja prevalencia. En otras especies de peces Haemulidos en Venezuela (Haemulon steindachneri (Jordan y Gilbert, 1882); H. aurolineatum Cuvier, 1830; H. chrysargyreum Günther, 1859), se ha observado que los monogeneos fueron dominantes en la comunidad parasitaria, destacando la especie cogenérica Choricotyle aspinachorda Hargis, 1955 (Centeno y Bashirullah, 2003).

González et al. (2001) observaron a Choricotyle sp como la dominante entre los ectoparásitos en I. conceptionis, con un $13.9 \%$ de prevalencia, similar al observado en el presente estudio, en tanto que Oliva $e t$ al. (2009) señalan un 54\% de prevalencia para $C$. isaciencis en la Bahía de San Jorge, Chile. En el haemulido Anisotremus scapularis (Tschudi) se ha observado a Choricotyle anisotremi Oliva, 1987, en branquias y cavidad bucofaríngea con un 6.6\% de parasitismo en las costas peruanas (Iannacone y Alvariño, 2009a). Luque et al. (1993) encontraron una variación en la prevalencia de 3.1 a $11.1 \%$ para cuatro especies de Choricotyle en $O$. ruber en Brasil. Iannacone y Alvariño (2012) observaron una alta prevalencia del monogeneo Mexicana sp en el haemúlido A. scapularis.

Los parásitos encontrados en $I$. conceptionis señalan que los tres índices de agregación [Dispersión (ID), Discrepancia de Poulin (D) y Binomial Negativa (k)] mostraron mayormente una distribución sobredispersa para los parásitos. Se ha argumentado varios factores para que este tipo de distribución sea la más típica en los parásitos de peces (Iannacone et al., 2012).

La $\mathrm{P}$ de C. isaciencis estuvo correlacionada con la longitud total del hospedero. Opuestamente, González et al. (2001) encontraron que la Intensidad de Choricotyle sp estuvo correlacionada negativamente con la talla de I. conceptionis. Varias especies de helmintos de fauna 
ictiológica marina del Perú señalan que la $\mathrm{P}$ presenta correlaciones negativas o positivas con la longitud total del hospedero (Iannacone y Alvariño, 2008; Iannacone et al., 2010a,b, 2011, 2012). En relación con el sexo de $I$. conceptionis, no se observó relación con la $\mathrm{P}$ y abundancia de infección. Estos resultados reiteran lo que pudiera señalar una ausencia de diferencias en el comportamiento trófico entre los peces hembra y macho de $I$. conceptionis (Centeno y Bashirullah, 2003; Iannacone y Alvariño, 2008).

Una característica encontrada durante el periodo de muestreo fue que la fauna de helmintos parásitos de I. conceptionis presentó tres formas larvarias: del cestodo Nybelinia sp, del acantocefalo C. obtuscens y del nematodo $P$. carvajali. Nybelinia sp es el género de mayor riqueza en especies entre los Tripanorhyncha (Palm, 1999; Khamkar, 2012). Shimazu (1999) señala que el conocimiento del ciclo biológico de los Tripanorhyncha es limitado, siendo los hospederos definitivos peces elasmobranquios. En el caso de Nybelinia, no se conoce la oncosfera y la larva procercoide. Los eufásidos son considerados hospederos intermediarios de Nybelinia, que albergan las formas plerocercoides, y los peces teleósteos como I. conceptionis son considerados hospederos paraténicos. Bryan et al. (2012) consideran que las variaciones en la distribución del zooplancton y de los peces ocasionan oscilaciones en el cestodo Nybelinia surmenicola Okada, 1929, en su hospedero teleósteo. Las larvas de Nybelinia presentan una amplia distribución geográfica y una baja especificidad a su hospedero (Magalhaes Pinto et al., 2010).

En el Perú, $C$. obtuscens tiene como hospedero definitivo al lobo marino chusco, león marino o lobo de un pelo, Otaria byronia (Shaw, 1800) (Tantaleán et al., 2005). Los estadios juveniles (acantelas y cistacantos) son encontrados en 17 especies de peces que actúan como paraténicos de importancia comercial en el Perú (Tantaleán et al., 2005; Laskowski y Zdzitowiecki, 2009; Iannacone y Alvariño, 2009b; Iannacone et al., 2011). La presencia de Corynosoma en el ecosistema de Chorrillos, Lima, Perú, parece estar relacionada con las relaciones tróficas entre los microcrustáceos, la especie parásita indicada, los peces paraténicos involucrados como I. conceptionis y los pinnípedos, hospederos definitivos (Aznar et al., 2006; Morgades et al., 2006; Braicovich et al., 2011; Hernández-Orts et al., 2011).

El rol de $P$. carvajali en $I$. conceptionis no está claro. Al parecer, podría ocurrir el mismo patrón de comportamiento que en $A$. scapularis (Iannacone y Alvariño, 2009). Al igual que en otros peces de la familia Haemulidae de América del Sur, la presencia de larvas de helmintos parásitos (cestoda y acantocéfala) indica que la cabinza tiene un rol en un nivel medio en la cadena trófica marina (Marcogliese, 2002; Mendoza-Cruz et al., 2013).

En relación con $\mathrm{k}_{\mathrm{n}}$, no se vio que los peces parasitados por $C$. obtuscens, $C$. isaciencis, ectoparásitos y endoparásitos presentaran valores más altos en comparación con los peces no parasitados. Algunos investigadores han encontrado una asociación entre el $\mathrm{K}_{\mathrm{n}}$ y el parasitismo por helmintos, mostrando un efecto en la salud del pez hospedero (Neff y Cargnelli, 2004; Iannacone y Alvariño, 2013). Silva et al. (2011) explicó que el $\mathrm{k}_{\mathrm{n}}$ es un indicador cuantitativo para detectar parásitos que afectan el bienestar de los peces hospederos. Variaciones en el $\mathrm{k}_{\mathrm{n}}$ indican cambios en el ambiente, carencia de alimento o parasitismo. Lo esperado es que peces más infectados presenten valores más bajos de $\mathrm{k}_{\mathrm{n}}$ (Graça et al., 2013). Moreira et al. (2010) explicaron que asociaciones positivas entre el $\mathrm{k}_{\mathrm{n}}$ y la abundancia y prevalencia de algunos parásitos podrían ser explicadas por la mayor abundancia de alimento en el tracto digestivo para los endoparásitos virulentos, más espacio físico disponible y más ingesta de alimento que podría contener hospederos intermediarios con formas infectivas. La independencia entre el crecimiento y ganancia de peso $\left(\mathrm{k}_{\mathrm{n}}\right)$ y el parasitismo en $I$. 
conceptionis podría deberse a la baja patogenicidad de los parásitos, que influencian muy poco la correlación $\mathrm{k}_{\mathrm{n}}$ /parasitismo (Moreira et al., 2010). Denny et al. (2013) encontraron carencia de asociación entre el parasitismo y el factor de condición de Anguilla rostrata Lesueur, 1821 (Anguillidae).

El IGS solo se correlacionó con la LT de la cabinza, encontrándose ausencia de relación entre la IGS con las parasitosis de $I$. conceptionis. Simková et al. (2005) encontraron un incremento de la parasitosis con el aumento del IGS con la abundancia de monogeneos, cestoda y digeneos en el pez Barbatula barbatula (Linnaeus, 1758) (Balitoridae), indicando que los peces son más susceptibles a la infección parasitaria en los periodos de mayor gasto reproductivo. Kesler et al. (2009) encontraron relaciones negativas entre el IGS y el parasitismo en Cobitis taenia Linnaeus, 1758 (Cobitidae). Denny et al. (2013) encontraron carencia de asociación entre el parasitismo y el IGS de $A$. rostrata.

Los índices de diversidad alfa $\left(\mathrm{H}^{\mathrm{I}}\right.$ y $\left.\mathrm{J}^{\mathrm{I}}\right)$ de la parasitofauna de I. conceptionis mostraron valores relativamente altos y los índices de dominancia $\left(\lambda\right.$ y $\left.\mathrm{I}_{\mathrm{B}-\mathrm{P}}\right)$ tuvieron valores bajos. Los índices de interactividad $\left(\mathrm{CC}_{50}\right)$ de ectoparásitos y endoparásitos mostraron valores bajos que muestran que las comunidades no son interactivas o son aisladas para una muestra de 123 ejemplares en solo cuatro meses de evaluación y un $38.2 \%$ de parasitismo. González et al. (2001) encontraron solo un $34 \%$ de parasitismo en $I$. conceptionis de Coquimbo, Chile, al evaluar 375 ejemplares a lo largo de un año, indicando una infracomunidad en este hospedero con una pobreza en número de especies, ausencia de especies dominantes (núcleo) y carencia de relaciones interespecíficas entre los parásitos. Este mismo patrón se observó para los ectoparásitos y endoparásitos de $A$. scapularis para las costas peruanas (Iannacone y Alvariño, 2009a). El estimador no paramétrico indicó que no se necesita in- crementar el esfuerzo de muestreo de $I$. conceptionis. Estos índices están influenciados y son más sensibles a las especies raras (Iannacone y Alvariño, 2013; Méndez, 2013).

\section{Conclusiones}

- Los cuatro parásitos con mayor prevalencia y abundancia media fueron Choricotyle isaciencis Oliva, González, Rus \& Luque, 2009, Corynosoma obtuscens (Lincicome, 1943), Proleptus carvajali Fernández y Villalba, 1985, y Clavella simplex Castro y Baeza, 1985.

- Los parásitos de I. conceptionis señalan que los tres índices de agregación [Dispersión (ID), Discrepancia de Poulin (D) y Binomial Negativa (k)] mostraron mayormente una distribución agregada.

- La prevalencia de C. isaciencis estuvo correlacionada con la longitud total del pez hospedero.

- En relación conl $\mathrm{k}_{\mathrm{n}}$ (factor de condición relativo), no se vio que los peces con $C$. obtuscens, C. isaciencis, parasitados con ectoparásitos y endoparásitos presentaran valores más altos en comparación con los peces no parasitados.

- El índice gonadosomático (IGS) no se relacionó con ninguno de los índices de ecología parasitaria.

- Los índices de equidad de la parasitofauna mostraron valores relativamente altos y los índices de dominancia fueron bajos.

- Los índices de interactividad $\left(\mathrm{CC}_{50}\right)$ de ectoparásitos y endoparásitos indican que las comunidades son aisladas.

\section{Literatura Citada}

\section{Aznar FJ, Pérez-Ponce de León G,}

Raga JA. 2006. Status of Corynosoma (Acantocephala: Polymorphidae) based on anatomical, ecological, and phylogenetic evidence, with the erection 
of Pseudocorynosoma n. gen. J Parasitol 93: 548-564. doi: 10.1645/GE715R.1

2. Bautista CE, Monks S, Pulido G 2013. Los parásitos y el estudio de su biodiversidad: un enfoque sobre los estimadores de la riqueza de especies. En: Pulido-Flores G, Monks C (eds). Estudios científicos en el estado de Hidalgo y zonas aledañas. Vol 2. Hidalgo, México: Universidad Autónoma del Estado de Hidalgo. p 13-17.

3. Bego NM, Von Zuben CJ. 2010. Métodos quantitativos em parasitologia. Jaboticabal: FUNEP. 72 p.

4. Bliss CI, Fisher, RA. 1953. Fitting the negative binomial distribution to biological data. Biometrics 9: 176-200.

5. Braicovich PE, González RA, Tanzola, RD. 2005. First record of Corynosoma australe (Acanthocephala, Polymorphidae) parasitizing seahorse, Hippocampus sp (Pisces, Syngnathidae) in Patagonia (Argentina). Acta Parasitol 50: 145-149.

6. Bryan DR, Jacobson KC, Buchanan, JC. 2012. Recent increase in Nybelinia surmenicola prevalence and intensity in Pacific Hake (Merluccius productus) off the United States West Coast. J Parasitol 98: 85-92. doi: 10.1645/GE2906.1

7. Bush AO, Lafferty KD, Lotz JL, Shostak, AW. 1997. Parasitology meets ecology on its own terms: Margolis et al. revisited. J Parasitol 83: 575-583.

8. Castro RC, Baeza KH. 1985. Clavella simplex sp. nov. (Copepoda: Lernaeopodidae), a parasite of Isacia conceptionis (Pisces: Teleostei) in Northern Chile. Crustaceana 49: 173176.

9. Centeno L, Bashirullah AK, Alvarez ME, Alvarez R. 2002. Análisis comparativo de las comunidades de parásitos metazoarios en dos especies de peces marinos del Golfo de Cariaco, Venezuela. Bioagro 14: 135-144.

10. Centeno L, Bashirullah AK. 2003. Comunidades de parásitos metazoos en ocho especies de peces del género Haemulon (Fam: Haemulidae) del Golfo de Cariaco, Venezuela. Ciencia 11: 119-124.

11. Céspedes RE, Iannacone J, Salas A. 2011. Helmintos parásitos de Dosidicus gigas «Pota» eviscerada en Arequipa, Perú. Ecol Apl 10: 1-11.

12. Chirichigno FN, Vélez M. 1998. Clave para identificar los peces marinos del Perú. $2^{\text {a }}$ ed. Callao: Instituto del Mar del Perú. 500 p.

13. Chirichigno FN, Cornejo RM. 2001. Catálogo comentado de los peces marinos del Perú. Callao: Instituto del Mar del Perú, IMARPE. 314 p.

14. Cohen SC, Justo MCN, Kohn A. 2013. South American monogenoidea parasites of fishes, amphibians and reptiles. Rio de Janeiro: Conselho Nacional de Desenvolvimento Cientifico e Tecnologico (CNPq). 663 p.

15. Cortés N, Aron A. 2011. Ciclo reproductivo y fecundidad parcial de Isacia conceptionis (Perciformes, Haemulidae) en La Herradura, Coquimbo, Chile. Rev Biol Mar Oceanog 46: 101-104. doi: 10.4067/S071819572011000100016

16. Denny SK, Denny A, Tyson P. 2013. Distribution, prevalence and intensity of Anguillicoloides crassus in the American eel, Anguilla rostrata, in the Bras d'Or Lakes, Nova Scotia. BioInvasions Rec 2: 19-26. doi: 10.3391/ bir.2013.2.1.03

17. Esch GW, Shostak AW, Marcogliese DJ, Goater, TM. 1990. Patterns and process in helminth parasite communities: an overview. In: Esch G, Bush AC, Aho J (eds). Parasite communities: patterns and processes. New York: Chapman and Hall. p 1-19.

18. Felizardo NN, Torres EJL, Fonseca GMC, Pinto RM, Gomes CD, Knoff M. 2010. Cestodes of the flounder Paralichthys isosceles Jordan, 1890 (Osteichthyes Paralichthyidae) from the state of Rio de Janeiro, Brazil. Neotrop Helminthol 4: 113-125. 
19. González MT, Oliva EM, Muñoz C. 2001. Estacionalidad, tamaño corporal y sexo del hospedador como fuentes de variación de la ectoparasitofauna de la cabinza, Isacia conceptionis (Haemulidae) en el norte de Chile. En: IX Congreso Latinoamericano sobre Ciencias del Mar. Colombia.

20. Graça RJ, Costa APL, Takemoto RM. 2013. Ecological aspects of monogenea gill parasites (Platyhelminthes) from Hoplias aff. malabaricus (Bloch, 1794) (Pisces, Erythrinidae) in a neotropical floodplain. Neotrop Helminthol 7: 105-116.

21. Hernández-Orts JS, Crespo JA, Montero FE, Raga JA, Aznar FJ. 2011. Population structure of Corynosoma australe (Acantocephala: Polymorphidae) in paratenic fish hosts from Patagonia, Argentina. $8^{\text {th }}$ International Symposium on Fish Parasites. Viña del Mar, Chile.

22. Iannacone J, Alvariño, L. 2008. Influencia del tamaño y sexo de Peprilus medius (Peters) (Stromateidae: Perciformes) capturados en Chorrillos, Lima, Perú, sobre su comunidad parasitaria. Neotrop Helminthol 2: 62-71.

23. Iannacone J, Alvariño L. 2009a. Aspectos cuantitativos de la parasitofauna de Anisotremus scapularis (Tschudi) (Osteichthyies, Haemulidae) capturados por pesquería artesanal en Chorrillos, Lima, Perú. Rev Ibero-Latinoam Parasitol 1: 56-64.

24. Iannacone J, Alvariño L. 2009b. Dinámica poblacional de la diversidad parasitaria de la «cabrilla» Paralabrax humeralis (Teleostei: Serranidae) en Chorrillos, Lima, Perú. Neotrop Helminthol 3: 73-88.

25. Iannacone J, Morón L, Guizado S. 2010a. Variación entre años de la fauna de parásitos metazoos de Sciaena deliciosa (Tschudi, 1846) (Perciformes: Sciaenidae) en Lima, Perú. Lat Am J Aquat Res 38: 218-226. doi: 10.4067/ S0718-560X2010000200006
26. Iannacone J, Alvariño L, CárdenasCallirgos J. 2010b. Ecología comunitaria de los parásitos de la chilindrina Stromateus stellatus (Cuvier, 1829) (Perciformes: Stromateidae) de la zona costera de Chorrillos, Lima, Perú. Neotrop Helminthol 4: 159-167.

27. Iannacone J, Cerapio JP, CárdenasCallirgos J, Sánchez K, Briceño F, Dueñas A. 2011. Comunidades de parásitos en el trambollo Labrisomus philippii (Steindachner, 1866) (Perciformes: Labrisomidae) de la zona costera de Chorrillos Lima, Perú. Neotrop Helminthol 5: 73-84.

28. Iannacone, J, Alvariño L. 2012. Microecology of the monogenean Mexicana sp on the gills of Anisotremus scapularis (Tschudi, 1846) (Osteichthyes, Haemulidae) of the marine coast of Lima, Peru. Neotrop Helminthol 6: 277-285.

29. Iannacone J, Sánchez V, Olazábal N, Salvador C, Alvariño L, Molano J. 2012. Ecological indices of parasites of Scartichthys gigas (Steindachner, 1876) (Perciformes: Blenniidae) of the coasts of Lima, Peru. Neotrop Helminthol 6: 191-203.

30. Iannacone J, Alvariño L. 2013. Parasitological indices of Pacific pomfret Brama japonica Hilgendorf, 1878 (Osteichthyes, Bramidae) acquired at the fishing Terminal of Chorrillos, Lima, Peru. Neotrop Helminthol 7: 117-132.

31. [IMARPE] Instituto del Mar del Perú. 2014. "Cabinza» Isacia conceptionis (Cuvier and Valenciennes, 1830). [Internet]. Disponible en: http:// w w w i marpe.pe/i marpe/ ?id_especie $=000019$

32. Karling LC, Lacerda ACF, Takemoto RM, Pavanelli GC. 2013. Ecological relationships between endoparasites and the fish Salminus brasiliensis (Characidae) in a Neotropical floodplain. Neotrop Helminthol 7: 219-230. 
33. Kesler M, Vetemaa M, Saks L, Saat T. 2009. Occurrence of Ligula colymbi (Cestoda) in spinach loach (Cobitis taenia) and its effects on reproduction and growth of the host. Boreal Environ Res 14: 932-936.

34. Khamkar DD. 2012. A report of the species of the genus Nybelinia (Cestoda: Trypanorhyncha) from a Trygon Sepehn, from Panji, Goa state, India. Trends Parasitol Res 1: 31-33.

35. Kohn A, Fernandes BMM, Cohen SC. 2007. South American trematodes parasites of fishes. Rio de Janeiro: Conselho Nacional de Desenvolvimento Cientifico e Tecnologico (CNPq). 318 p.

36. Laskowski Z, Zdzitowiecki K. 2009. Occurrence of acanthocephalans in notothenioid fishes in the Beagle Channel (Magellanic sub-region, sub-Antarctic). Polish Polar Res 30: 179-186.

37. Longart RY, Acosta V, Parra B, Lista M. 2011. Aspectos biométricos de Hemirhamphus brasiliensis (Pisces: Hemirhamphidae), Isla de Cubagua, Venezuela. Zootecnia Trop 29: 385-398.

38. Luque JL, Farfán C. 1991. Neobrachiella kabatai n. sp. (Copepoda: Lernaeopodidae) parasitic on Isacia conceptionis (Cuvier) (Osteichthyes: Pomadasyidae) from Peruvian waters. Syst Parasitol 20: 135139. doi: 10.1007/BF00007389

39. Luque J, Amato JFR, Takemoto RM. 1993. Four species of Choricotyle Van Beneden \& Hesse (Monogenea: Diclidophoridae: Choricotylinae) parasitic on Orthopristis ruber (Cuvier) (Osteichthyes: Haemulidae) from the Brazilian coast, with description of two new species. Rev Bras Parasitol Vet 2: $15-24$.

40. Luque JL, Poulin R. 2008. Linking ecology with parasites diversity in Neotropical fishes. J Fish Biol 72: 189204. doi:10.1111/j.1095-8649.2007. 01695.x

41. Marcogliese D. 2002. Food webs and the transmission of parasites to marine fish. Parasitology 124: 83-99.
42. Méndez O. 2013. Diversidad de helmintos de peces dulceacuícolas en los oasis de Baja California Sur, México. Biologist (Lima) 11: 287-305.

43. Mendoza-Cruz M, Valles-Vega I, Lozano-Cobo H, Gómez del Prado-Rosas MC, Castro-Moreno PN. 2013. Parasitefauna of Paranthias colonus (Valenciennes, 1846) from el Sargento, Baja California Sur, México. Neotrop Helminthol 7: 13-28.

44. Morales GE, Sarmiento BL, Rivera TG. 1987. Parásitos de Isacia conceptionis »cabinza» y Stellifer minor «mojarrilla» de la Reserva de Paracas. En: Resúmenes X Congreso Latinoamericano de Microbiología. Trujillo, Perú. p 77.

45. Moreno E. 2001. Métodos para medir la biodiversidad. M\&T - Manuales y Tesis SEA. Cooperación Iberoamericana CYTED. UNESCO Orcyt. México: Sociedad Entomológica Aragonesa. 84 p.

46. Moreira LHA, Yamada FH, Ceschini TL, Takemoto RM, Pavanelli C. 2010.

The influence of parasitism on the relative condition factor $(\mathrm{Kn})$ of Metynnis lippincottianus (Characidae) from two aquatic environments: the upper Parana river floodplain and Corvo and Guairacá rivers, Brazil. Acta Sci Biol Sci 32: 8386. doi: 10.4025/actascibiolsci.v32i1.3668

47. Morgades D, Katz H, Castro O, Capellino D, Casas L, Benitez G, Venzal JM, Moraña A. 2006. Fauna parasitaria del lobo fino Arctocephalus australis y del león marino Otaria flavescens (Mammalia, Otariidae) en la costa uruguaya. En: Menafra R, Rodríguez-Gallego L, Scarabino F, Conde D (eds). Bases para la conservación y el manejo de la costa uruguaya. Montevideo, Uruguay: Vida Silvestre. p 89-96.

48. Muñoz G Olmos V. 2007. Revisión bibliográfica de especies ectoparásitas y hospedadoras de sistemas acuáticos de Chile. Rev Biol Mar Oceanog 42: 89148 . do i : 10.4067 / S 0718 19572007000200001 
49. Muñoz G Olmos V. 2008. Revisión bibliográfica de especies endoparásitas y hospedadoras de sistemas acuáticos de Chile. Rev Biol Mar Oceanog 43: 173-245. doi: 10.4067/S0718-19572008000200002

50. Neff BD, Cargnelli LM. 2004. Relationships between condition factors, parasite load and paternity in bluegill sunfish, Lepomis macrochirus. Environ Biol Fish 71: 297-304. doi: 10.1007/ s10641-004-1263-8

51. Oliva ME, González MT, Ruz PM, Luque JL. 2009. Two new species of Choricotyle Van Beneden \& Hesse (Monogenea: Diclidophoridae), parasites from Anisotremus scapularis and Isacia conceptionis (Haemulidae) from northern Chilean coast. J Parasitol 95: 1108-1111. doi: 10.1645/GE-2038.1

52. Palm H. 1999. Nybelinia Poche, 1926 , Heteronybelinia gen. nov. and Mixonybelinia gen. nov. (Cestoda, Trypanorhyncha) in the collections of The Natural History Museum, London. Bull Nat Hist Mus London 65: 133-153.

53. Poulin R. 1993. The disparity between observed and uniform distributions: a new look at parasite aggregation. Int $\mathbf{J}$ Parasitol 23: 937-944.

54. Poulin R, Luque JL. 2003. A general test of the interactive-isolationist continuum in gastrointestinal parasite communities of fish. Int J Parasitol 33: 1623-1630. doi: 10.1016/j.ijpara.2003.09.005

55. Poulin R. 2010. Latitudinal gradients in parasite diversity: bridging the gap between temperate and tropical areas. Neotrop Helminthol 4: 169-177.

56. Reiczigel J. 2003. Confidence intervals for the binomial parameters: some new considerations. Stat Med 22: 611-621. doi: 10.1002/sim. 1320

57. Rodhe K, Hayward C, Heap M. 1995. Aspects of the ecology of metazoan ectoparasites of marine fishes. Int $\mathbf{J}$
Parasitol 25: 945-970. doi: 10.1016/00207519(95)00015-T

58. Rózsa L, Reiczigel J, Majoros G. 2000. Quantifying parasites in samples of hosts. J Parasitol 86: 228-232.

59. Sarmiento L, Tantaleán M, Huiza A. 1999. Nematodos parásitos del hombre y de los animales en el Perú. Rev Peru Parasitol 14: 9-65.

60. Shimazu T. 1999. Plerocercoids with blastocystics of the Trypanorhynch cestode Nybelinia surmenicola found in the euphausiid crustacean Euphasia pacifica. Otsuchi Mar Sci 24: 1-4.

61. Silva OAM, Tavares-Dias M, Fernandes, JS. 2011. Helminthes parasitizing Semaprochilodus insignis Jardine, 1841 (Osteichthyes: Prochilodontidae) from the central Amazonia (Brazil), and their relationship with the host. Neotrop Helminthol 5: 225-233.

62. Simková A, Jarkovský J, Koubková B, Barus V, Prokes M. 2005. Associations between fish reproductive cycle and the dynamics of metazoan parasite infection. Parasitol Res 95: 6572. doi: 10.1007/s00436-004-1261-y

63. Tantaleán M, Carvajal G, Martínez R, Huiza A. 1982. Helmintos parásitos de peces marinos de la costa peruana. Lima, Perú: Naturaleza, Ciencia y Tecnología local para el Servicio Social NCTL. 40 p.

64. Tantaleán M, Huiza A. 1994. Sinopsis de los parásitos de peces marinos de la costa peruana. Biotempo 1: 53-101.

65. Tantaleán M, Sánchez L, Alina $H$. 2005. Acantocéfalos del Perú. Rev Per Biol 12: 83-92.

66. Tantaleán M, Sarmiento L, Huiza, A. 1992. Digeneos (Trematoda) del Perú. Bol Lima (Perú) 80: 47-84.

67. Vargas MF, Cifuentes PS, Emparanza ME. 1999. Espectro trófico de peces concurrentes al área de crianza Playa Chipana (21 $\left.{ }^{\circ} 19^{\prime} \mathrm{S}-70^{\circ} 04^{\prime} \mathrm{W}\right)$ del norte de Chile. Rev Biol Trop 47: 597-600. 